Supporting Information for:

\title{
Subpercent Local Strains due to the Shapes of Gold Nanorods Revealed by Data-Driven Analysis
}

\author{
Kohei Aso, ${ }^{*}, 1,2$ Jens Maebe, ${ }^{3}$ Xuan Quy Tran, ${ }^{2}$ Tomokazu Yamamoto, ${ }^{4}$ Yoshifumi Oshima, ${ }^{1}$ \\ and Syo Matsumura ${ }^{2,4}$
}

${ }^{1}$ School of Materials Science, Japan Advanced Institute of Science and Technology, Asahidai 1-1, Nomi, Ishikawa, 923-1292, Japan

${ }^{2}$ Department of Applied Quantum Physics and Nuclear Engineering, Kyushu University, Motooka 744, Nishi-ku, Fukuoka 819-0395, Japan

${ }^{3}$ Faculty of Sciences, Ghent University, Krijgslaan 281-S2, 9000 Ghent, Belgium

${ }^{4}$ The Ultramicroscopy Research Center, Kyushu University, Motooka 744, Nishi-ku, Fukuoka 819-0395, Japan

*Corresponding author e-mail: aso@jaist.ac.jp

\section{Imaging of a standard sample $\mathrm{SrTiO}_{3}$}

During acquisition of a high-angle annular dark-field (HAADF) image in scanning transmission electron microscopy (STEM), a focused electron probe is scanned across an area of interest in the sample, and scattered electrons are collected by the annular detector point-bypoint to form a final image. Such the image forming process of STEM often causes image distortion due to electrical noises and local scanning disorder due to positional probe instability. HAADF-STEM imaging of $\mathrm{SrTiO}_{3}$ with a perovskite-type cubic structure was analyzed to evaluate the image distortion inherent in JEM-ARM200F ACCELARM operated at the same condition as at the main experiment. 
A thin film of $\mathrm{SrTiO}_{3}$ was used as a standard sample for the estimation of image distortion. The thin sample was cut from a $\mathrm{SrTiO}_{3}$ bulk by using a focused ion beam (FIB) instrument (Quanta 3D 200i, FEI, US). The sample was then thinned down to less than $100 \mathrm{~nm}$ by Ar ion beam milling (PIPS II, Gatan, US), where the acceleration voltage of the Ar ion beam was gradually lowered down from $5 \mathrm{keV}$ to $1 \mathrm{keV}$, and finally $0.3 \mathrm{keV}$ to remove contamination and damages.

Examples of HAADF-STEM images of $\mathrm{SrTiO}_{3}$ in [001] orientation are shown in Figure $\mathrm{S} 1$. The images are acquired with scanning angles $\theta=0^{\circ}, 90^{\circ}, 180^{\circ}$, and $270^{\circ}$ relative to the default scan direction, and each image is the integration of 10 frames with a scanning speed of 1 $\mu$ s/pixel. The scanning direction can be divided into fast scan and orthogonal slow scan directions. In the former direction, two adjacent pixels in an image are continuously acquired. After the acquisition of all pixels in the fast scan direction, the probe shifts along the slow scan direction, then the image is acquired again along the fast scan direction. Therefore, two adjacent pixels in the slow scan direction are acquired with slower intervals dependent on image size, compared to the fast scan direction. The local image distortion is often caused by the difference in acquisition interval between the fast and slow scan directions. The fast scan and the slow scan directions are indicated as green and red arrows in Figure S1.

As HAADF image intensity depends on atomic number $Z$ as $Z^{\sim 1.7}$, brighter and darker blobs correspond to [001] atomic columns of strontium ( $\mathrm{Sr}$ ) and mixed ones of titanium (Ti) and oxide (O) atoms. Atomic columns of pure $\mathrm{O}$ atoms were not discernable in these images. Similar sets of HAADF-STEM images were obtained in different magnifications and pixel sizes of $512 \times$ 512, $1024 \times 1024$, and $2048 \times 2048$, corresponding to the conditions for Au nanoparticles of NP1.0, NP2.1, and NP3.6. 

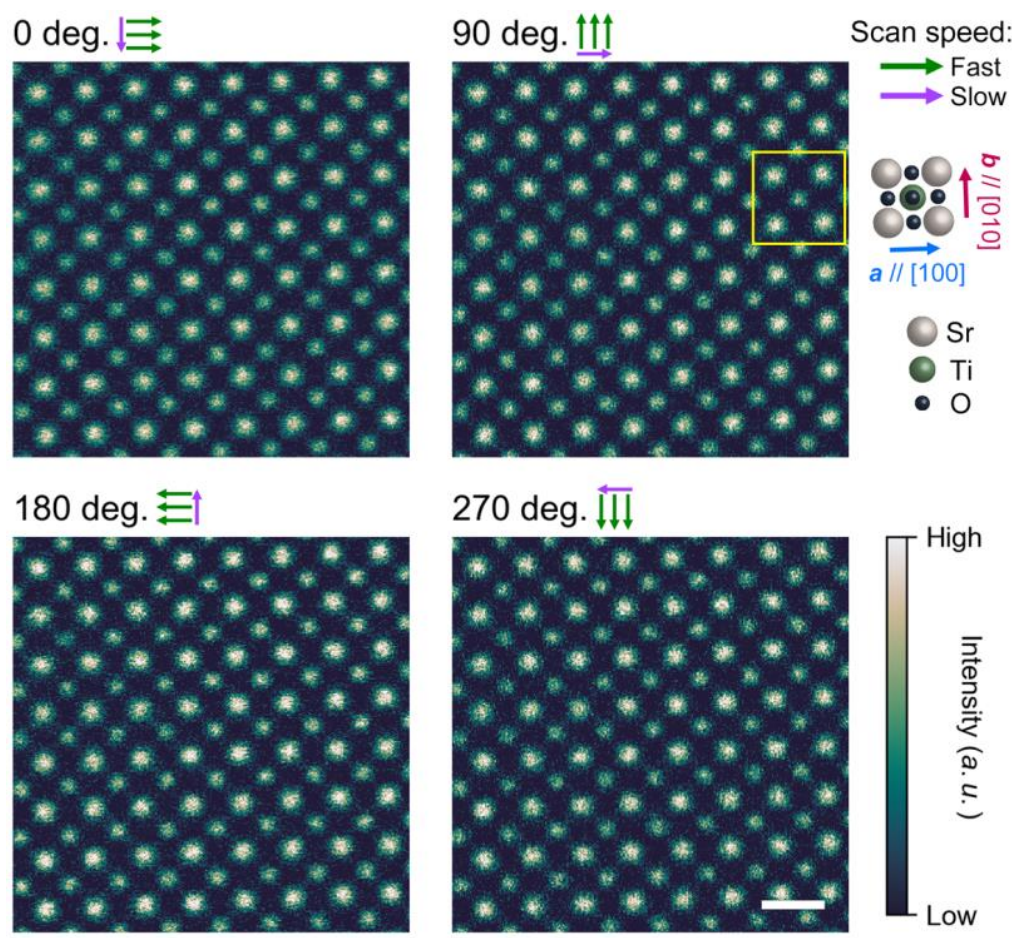

Figure S1. Examples of atomic resolution images of $\mathrm{SrTiO}_{3}$ taken by different scanning directions. The sewing directions are indicated by green arrows above the images. The scale bar in the lower right is $500 \mathrm{pm}$.

\section{Evaluation and correction of systematic distortions in STEM imaging 1,2}

Figures S2a and S2b show histograms of unit cell vectors $\boldsymbol{d}_{100}$ and $\boldsymbol{d}_{010}$ in magnitude and in interior angle, respectively, measured in an original HAADF image of [001] $\mathrm{SrTiO}_{3}$ for $\theta=$ $0^{\circ}$. The distributions of magnitudes of $d_{100}$ and $d_{010}$ are almost overlapped to each other, but the peak positions are slightly shifted to be smaller from the exact lattice parameter of $a_{0}=390.5$ pm for $\mathrm{SrTiO}_{3},{ }^{3}$ as seen in Figures S2a. The interior angles between $\boldsymbol{d}_{100}$ and $\boldsymbol{d}_{010}$ distribute symmetrically around $91.3^{\circ}$ larger than the right angle in Figure S2b. The similar measurements on $\boldsymbol{d}_{110}$ and $\boldsymbol{d}_{\overline{110}}$ are summarized in Figures S2c and 2d, where the histograms of equivalent $d_{110}$ and $d_{\overline{1} 10}$ appear mismatched to each other but the angle distribution draws a peak at $89.8^{\circ}$ close to the exact angle. Figure S2a-S2d clearly characterizes the image distortion included in the original HAADF-STEM image due to STEM equipment. 
The image distortions were corrected by the affine transformation proposed by Sato et al. ${ }^{1,2}$ The results after correction are demonstrated in Figure S2e-S2h. The histograms of $d_{100}$ and $d_{010}$ values are coincided with each other in symmetrical forms with the averaged ones $\bar{d}_{100}=$ $\bar{d}_{010}=390.5 \mathrm{pm}$, and the angles between $\boldsymbol{d}_{100}$ and $\boldsymbol{d}_{010}$ are distributed around a peak at the exact angle of $90^{\circ}$ as shown in Figures S2e and S2f. The distributions of $\boldsymbol{d}_{110}$ and $\boldsymbol{d}_{\overline{1} 10}$ in Figures S2g and S2h characterize $\bar{d}_{110}=\bar{d}_{1 \overline{1} 0}=550.6 \mathrm{pm}(=\sqrt{2} a)$ and $\boldsymbol{d}_{110} \perp \boldsymbol{d}_{\overline{1} 10}$. The cubic symmetries of $\mathrm{SrTiO}_{3}$ are successfully confirmed after the correction of image distortion by the affine transformation. The affine matrices for distortion correction were prepared according to image acquisition conditions for HAADF-STEM images of the gold nanoparticles given in Figure 1.
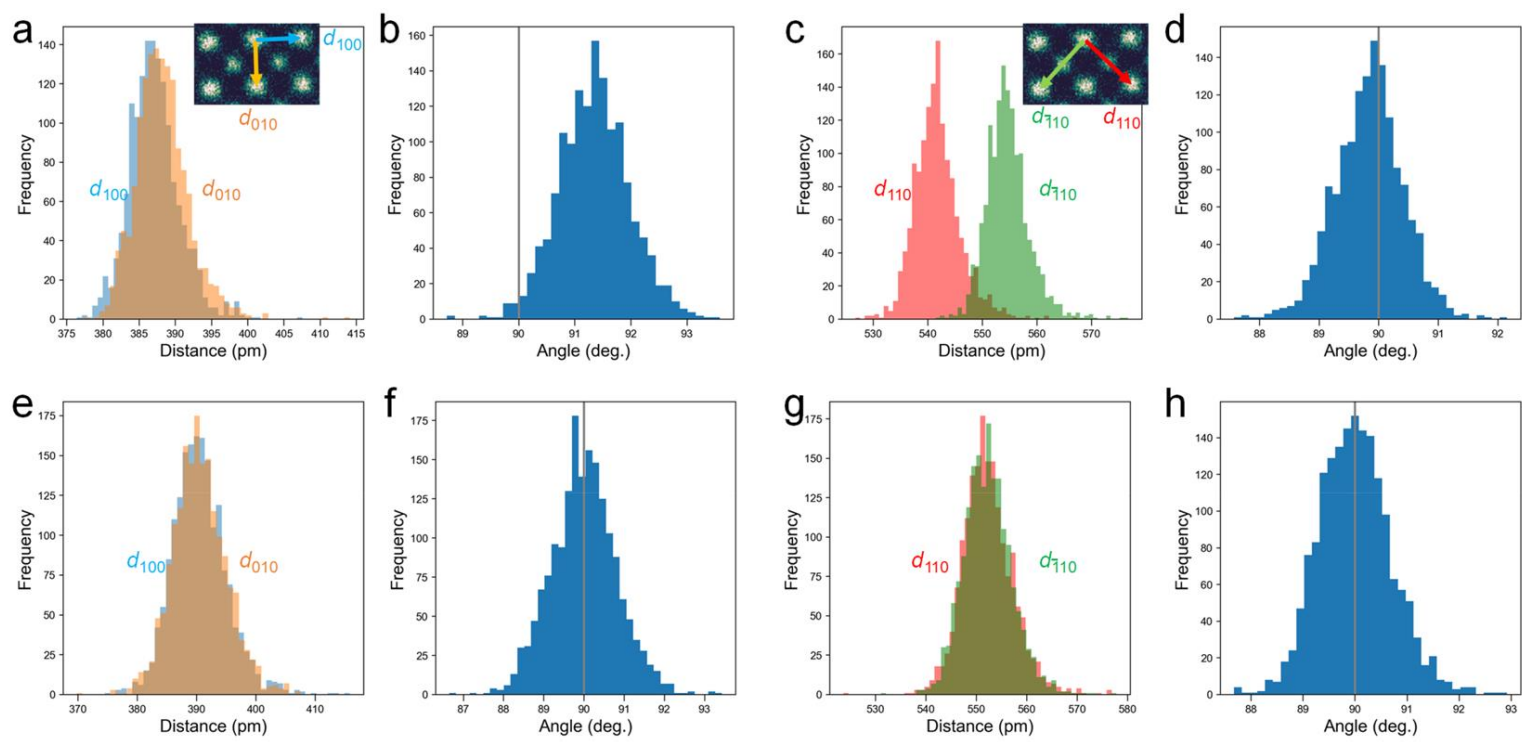

Figure S2. Histograms of structural parameters measured in a HAADF image of $\mathrm{SrTiO}_{3}$. Interatomic distances of $d_{100}$ and $d_{010}$ (a) and their angles (b), distances $d_{110}$ and $d_{\overline{1} 10}$ (c) and the angles (d). (e)-(h) show the corrected distributions correspondingly.

\section{Mathematics of Gaussian process regression (GPR) ${ }^{4,5}$}

GPR is a method to predict a true form of data with noise by using a framework of Bayesian statistics. The main purpose of this chapter is that GPR evaluates the true form as a summation 
of weighted continuous functions in a statistically reasonable approach. Let us suppose that we observe data consist of a scalar value $t$ at a position $\mathbf{r}$, where the scalar $t$ includes Gaussian white noise. We obtain the dataset including the scalars $\left(t_{1}, \cdots, t_{i}, \cdots, t_{f}\right)$ at the positions $\left(\mathbf{r}_{1}, \cdots, \mathbf{r}_{i}, \cdots, \mathbf{r}_{f}\right)$, where $i$ means the index, and $f$ means the end index of the dataset. The scalar sets are vectorized as $\mathbf{t}=\left(t_{1}, \cdots, t_{i}, \cdots, t_{f}\right)^{T}$ for the following mathematical treatment. In this study, $t_{i}$ correspond to a displacement component $t_{i}=u_{x}\left(\right.$ or $\left.t_{i}=u_{y}\right)$ at an atomic position $\mathbf{r}_{i}=\left(x_{m}, y_{n}\right)$

GPR assumes the similarity of the values according to the distance between the observed positions. The similarity of any two pair in the dataset is given by a kernel function $k\left(\mathbf{r}_{g}, \mathbf{r}_{h}\right)$, where the indices $g$ and $h$ describe any pair of the data as an example. In this study, we used a squared-exponential function

$$
k\left(\mathbf{r}_{g}, \mathbf{r}_{h}\right)=a \exp \left(-\frac{\left\|\mathbf{r}_{g}-\mathbf{r}_{h}\right\|^{2}}{2 b^{2}}\right)
$$

as the kernel function, where $a$ and $b$ are hyperparameters. The squared-exponential kernel gives a higher similarity when the distance between the two points $\mathbf{r}_{g}$ and $\mathbf{r}_{h}$ have closer distance. It reflects an assumption that if the two points $\mathbf{r}_{g}$ and $\mathbf{r}_{h}$ are close, the values $t_{g}$ and $t_{h}$ should take also close value in the true form of data without noise. The hyperparameters $a$ and $b$ in the kernel function can be optimized to be the parameters that maximize the loglikelihood function

$$
p_{\ln }(b, c)=-\ln \left|\mathbf{C}_{f}\right|-\mathbf{t}^{T} \mathbf{C}_{f}^{-1} \mathbf{t}
$$

in a framework of Bayesian statistics. Here, $\mathbf{C}_{f}$ is the covariance matrix defined as 


$$
\mathbf{C}_{f}=\left(\begin{array}{cccc}
k\left(\mathbf{r}_{1}, \mathbf{r}_{1}\right) & k\left(\mathbf{r}_{1}, \mathbf{r}_{2}\right) & \cdots & k\left(\mathbf{r}_{1}, \mathbf{r}_{f}\right) \\
k\left(\mathbf{r}_{2}, \mathbf{r}_{1}\right) & k\left(\mathbf{r}_{2}, \mathbf{r}_{2}\right) & \cdots & k\left(\mathbf{r}_{2}, \mathbf{r}_{f}\right) \\
\vdots & \vdots & \ddots & \vdots \\
k\left(\mathbf{r}_{f}, \mathbf{r}_{1}\right) & k\left(\mathbf{r}_{f}, \mathbf{r}_{2}\right) & \cdots & k\left(\mathbf{r}_{f}, \mathbf{r}_{f}\right)
\end{array}\right)+s_{n}^{2}\left(\begin{array}{cccc}
1 & 0 & \cdots & 0 \\
0 & 1 & \cdots & 0 \\
\vdots & \vdots & \ddots & \vdots \\
0 & 0 & \cdots & 1
\end{array}\right)
$$

where $s_{n}^{2}$ is the variance of the noise level. In this study, the noise level $s_{n}$ is set to be the precision to determine atom positions in our experiments (from 2.3 to $4.0 \mathrm{pm}$ ).

With the optimized hyperparameters, GPR can estimate the true mean value without noise to be

$$
\operatorname{mean}\left(\mathbf{r}_{f+1}\right)=\mathbf{k}^{\mathrm{T}} \mathbf{C}_{f}^{-1} \mathbf{t}
$$

at a point $\mathbf{r}_{f+1}$. Note that we can select $\mathbf{r}_{f+1}$ from any one of the input positions $\left(\mathbf{r}_{1}, \cdots, \mathbf{r}_{i}, \cdots, \mathbf{r}_{f}\right)$. This case corresponds to the estimation of noise-free value at the selected input position. Equation S4 can be rewritten as a function of $\mathbf{r}_{f+1}$,

$$
\operatorname{mean}\left(\mathbf{r}_{f+1}\right)=\sum_{i=1}^{f} w_{i} k\left(\mathbf{r}_{i}, \mathbf{r}_{f+1}\right)
$$

where $w_{i}$ is a weight that is defined to be $\left(w_{1}, \cdots, w_{i}, \cdots, w_{f}\right)^{T}=\mathbf{C}_{f}^{-1} \mathbf{t}$. The mean value is clearly expressed as a weighted sum of the squared-exponential kernel functions. Because the kernel functions are continuous as expressed in Equation S1, we can obtain the derivative of the estimated mean value analytically. This feature of GPR enables us to estimate strain via the differentiation of the predicted displacement component. GPR can also provide the variance

$$
\operatorname{var}\left(\mathbf{r}_{f+1}\right)=c-\mathbf{k}^{\mathrm{T}} \mathbf{C}_{f}^{-1} \mathbf{k}
$$

where the vector $\mathbf{k}=\left(k\left(\mathbf{r}_{1}, \mathbf{r}_{f+1}\right), k\left(\mathbf{r}_{2}, \mathbf{r}_{f+1}\right), \cdots, k\left(\mathbf{r}_{f}, \mathbf{r}_{f+1}\right)\right)$, and the scalar $c=$ $k\left(\mathbf{r}_{f+1}, \mathbf{r}_{f+1}\right)+s_{n}^{2}$. The evaluated variance helps to judge whether the trends seen in the predicted values are statistically significant or not. The variance of the derivative can be also 
calculated, ${ }^{6}$ which provides the error in the estimation of the strain in this study. For example, 95\% confidence bands are shown for the experimental plots in Figure $3 \mathrm{~d}$ and $4 \mathrm{~d}$ in the main. For more details, the references [4] and [5] would be helpful.

\section{GPR processed displacements in gold nanoparticles}

Figures S3a and S3b show two-dimensional maps of raw data of displacements along the $x$-direction $u_{x}^{\text {raw }}\left(x_{m}, y_{n}\right)$ and the GPR processed displacements $\hat{u}_{x}^{\mathrm{GPR}}(x, y)$ in the three nanoparticle samples. On the other hand, raw data of displacements along the $y$ direction $u_{y}^{\text {raw }}\left(x_{m}, y_{n}\right)$ and the GPR processed displacements $\hat{u}_{y}^{\mathrm{GPR}}(x, y)$ are plotted in 2D maps in Figures S4a and S4b. As discussed in the main text, one may again confirm that GPR has successfully removed the random noise components included in the raw data of $u_{x}^{\text {raw }}\left(x_{m}, y_{n}\right)$ and $u_{y}^{\text {raw }}\left(x_{m}, y_{n}\right)$ for the three sample nanoparticles, and has disclosed proper displacement fields with smooth variations as $\hat{u}_{x}^{\mathrm{GPR}}(x, y)$ and $\hat{u}_{y}^{\mathrm{GPR}}(x, y)$ without any singularities due to over-fitting or under-fitting. In Figures S3 and S4, the results of MD simulations are also illustrated for comparison. The details of MD simulations are described later in SI\#6. The displacement fields of $\hat{u}_{x}^{\mathrm{GPR}}(x, y)$ and $\hat{u}_{y}^{\mathrm{GPR}}(x, y)$ look in good agreement with the simulations of MDrod models, in which the real shapes and dimensions of the sample nanoparticles were assumed. Significant displacements occur mostly in the tip end portions of rod-shaped particles, as demonstrated in Figures S3 and S4. Inward displacements to $x$-direction are found in the tip parts of ellipsoidal MDsph models, in an opposite sense to outward displacements in the experimental $\hat{u}_{x}^{\mathrm{GPR}}(x, y)$ and in the MDrod models. The local strains in the nanoparticles were evaluated as $e_{x x}(x)=\partial \hat{u}_{x}^{\mathrm{GPR}}(x, y) / \partial x$ and, $e_{y y}(y)=\partial \hat{u}_{y}^{\mathrm{GPR}}(x, y) /$ $\partial y$, taking advantage of a programming code developed by Hines et al. ${ }^{7}$ 


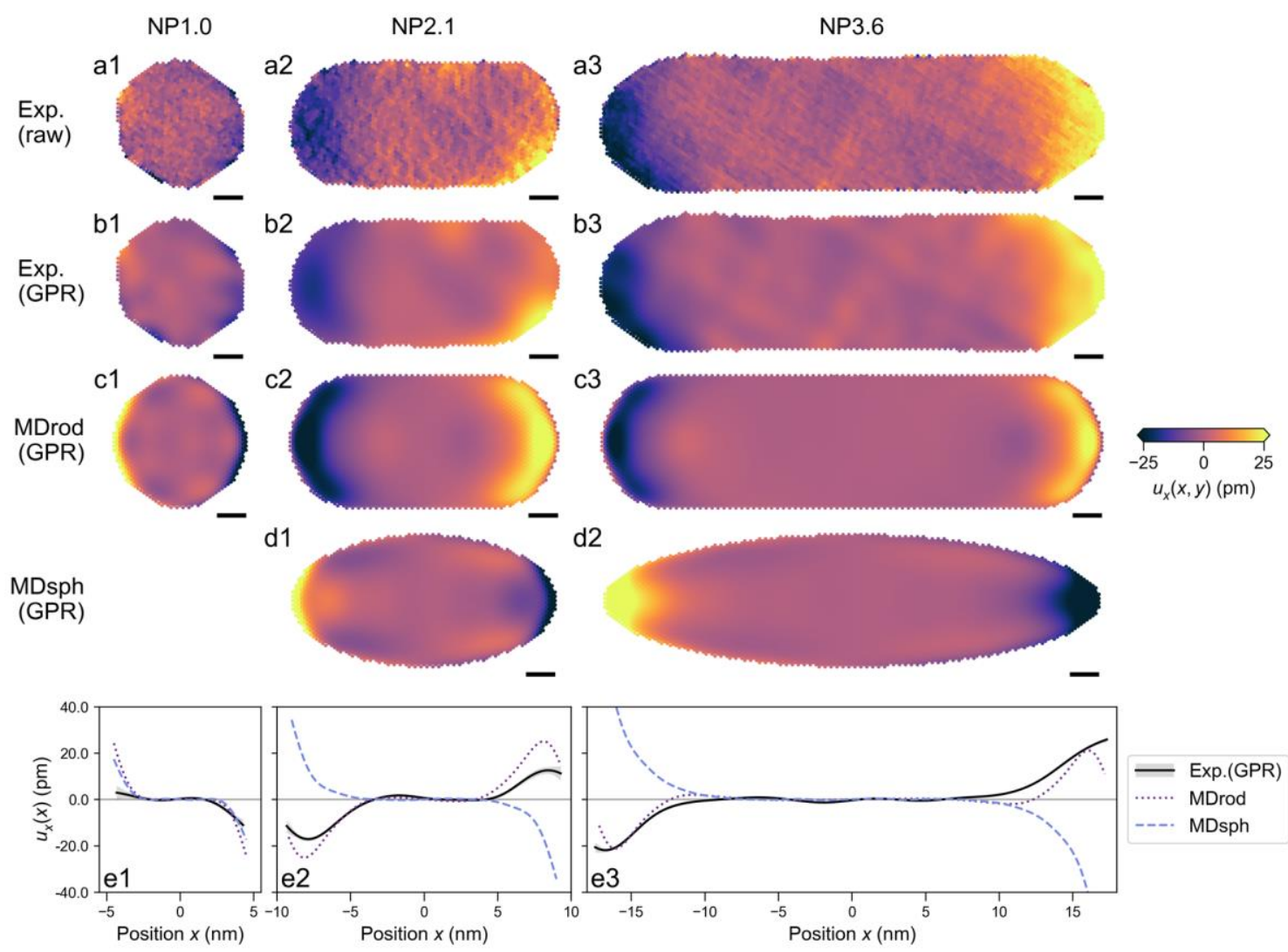

Figure S3. Displacement maps of $u_{x}(x, y)$ in the three sample nanoparticles. Original raw data $u_{x}^{\text {raw }}\left(x_{m}, y_{n}\right)$ (a). GPA processed $\hat{u}_{x}^{\mathrm{GPR}}(x, y)$ (b), MD simulations of MDrod models (c), and of MDsph models (d). The scale bars below the maps indicate $2 \mathrm{~nm}$. In (e), onedimensional line profiles of averaged $\bar{u}_{x}(x)$ are illustrated. 


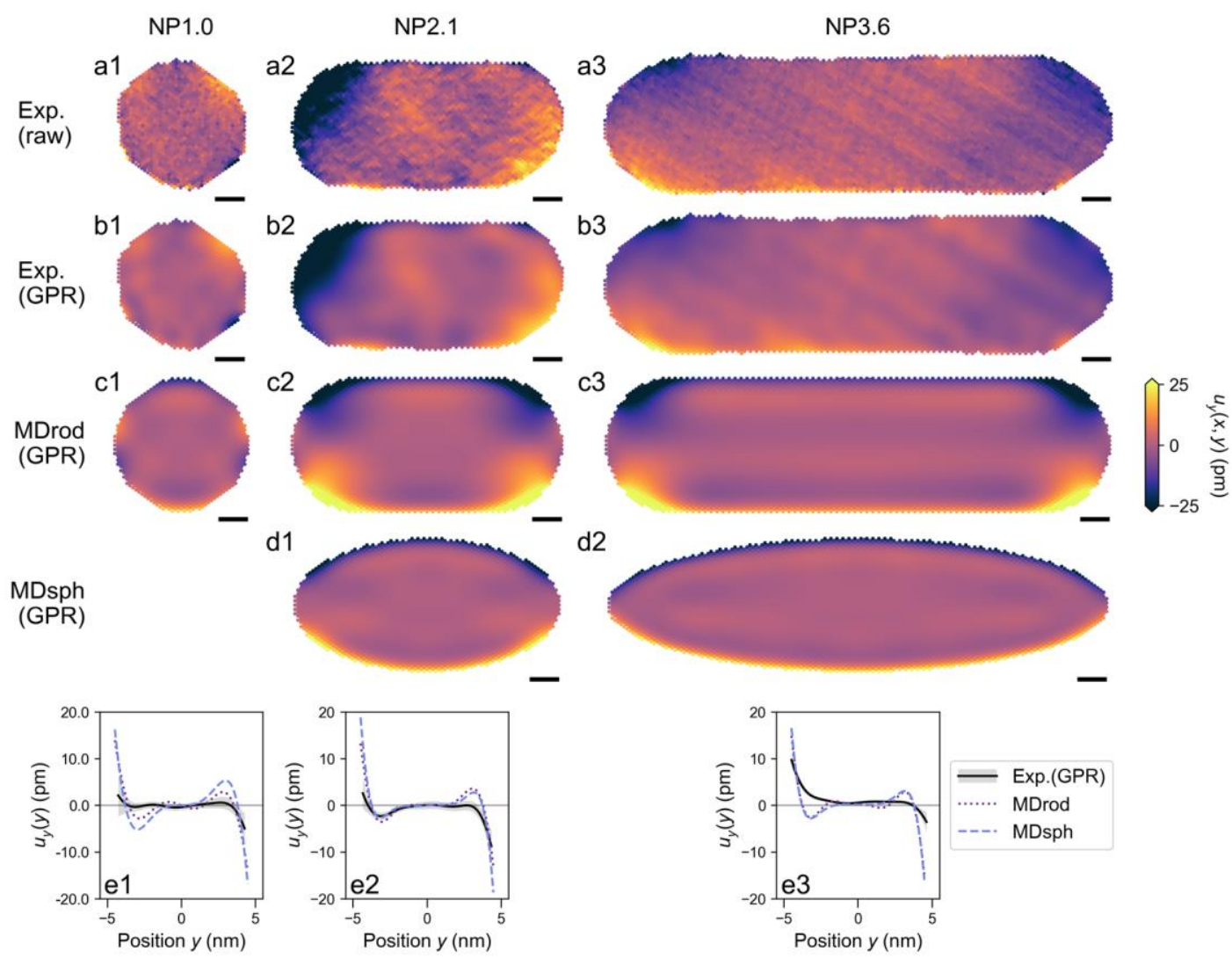

Figure S4. Displacement maps of $u_{y}(x, y)$ in the three sample nanoparticles. Original raw data $u_{y}^{\text {raw }}\left(x_{m}, y_{n}\right)(\mathbf{a})$. GPA processed $\hat{u}_{\boldsymbol{y}}^{\mathrm{GPR}}(x, y)$ (b), MD simulations of MDrod models (c), and of MDsph models (d). The scale bars below the maps indicate $2 \mathrm{~nm}$. In (e), onedimensional line profiles of averaged $\bar{u}_{y}(y)$ are illustrated.

\section{Local scanning distortion and its influence on strain measurements}

The local scanning disorder and its influence on local strain measurements on our instrument JEM-ARM200F ACCELARM were evaluated using [001] HAADF-STEM images of the same region of interest in a $\mathrm{SrTiO}_{3}$ sample acquired with different sewing directions of $\theta=0, \pi / 4$, $\pi / 2,3 \pi / 4$ as shown in Figure $\mathrm{S} 1$. The default scanning direction is $\theta=0$ for the acquisition of the images given in the main text. We first measured displacements $u_{x}\left(x_{m}, y_{n}, \frac{\pi}{4} h\right)$ and $u_{y}\left(x_{m}, y_{n}, \frac{\pi}{4} h\right)$ for every atomic column position from the periodic lattice site in the images, and then obtained the weighted averages of the displacement 


$$
\begin{aligned}
\hat{u}_{x \text { or } y}\left(x_{m}, y_{n}\right) & =\sum_{h=0}^{3} w_{x \text { or } y}\left(x_{m}, y_{n}, \frac{\pi}{4} h\right) u_{x}\left(x_{m}, y_{n}, \frac{\pi}{4} h\right) \\
& / \sum_{h=0}^{3} w_{x} \text { or } y\left(x_{m}, y_{n}, \frac{\pi}{4} h\right)
\end{aligned}
$$

Here, the statistical weight is defined as $w_{x}$ or $y\left(x_{m}, y_{n}, \frac{\pi}{4} h\right)=1 / \mid u_{x}$ or $y\left(x_{m}, y_{n}, \frac{\pi}{4} h\right)-$ $\left.\bar{u}_{x \text { or } y}\left(x_{m}, y_{n}\right)\right|^{2}$ with $\bar{u}_{x \text { or } y}\left(x_{m}, y_{n}\right)=\left(\sum_{h=0}^{3} u_{x \text { or } y}\left(x_{m}, y_{n}, \frac{\pi}{4} h\right)\right) / 4$ The deviations $D_{x \text { or } y}\left(x_{m}, y_{n}\right)=u_{x \text { or } y}\left(x_{m}, y_{n}, 0\right)-\hat{u}_{x \text { or } y}\left(x_{m}, y_{n}\right)$ are considered to give a measure of local image distortion due to the probe scanning disorder for $\theta=0$. The influence on local strain measurements was visualized by mapping of $E_{x x \text { or } y y}(x, y)$ which is the derivative of GPR processed $D_{x}$ or $y(x, y)$ as $E_{x x}(x, y)=\partial D_{x}(x, y) / \partial x$ and $E_{y y}(x, y)=\partial D_{y}(x, y) / \partial y$

Figure S5 maps $E_{x x}(x, y)$ and $E_{y y}(x, y)$ for imaging magnifications and sizes of $\times 15 \mathrm{M}$ and $(512)^{2}$ pixels $^{2}(\mathrm{a}), \times 12 \mathrm{M}$ and $(1024)^{2}$ pixels $^{2}(\mathrm{~b})$, and $\times 6 \mathrm{M}$ and $(2048)^{2}$ pixels $^{2}(\mathrm{c})$. As the white scale bars at the right bottom corners indicate $2 \mathrm{~nm}$, one may recognize enlargement of the imaging area with the pixel size. Stripe patterns faintly appear in the maps, being perpendicular to each between $E_{x x}(x, y)$ and $E_{y y}(x, y)$. It suggests that the positional differentiation accentuates the scanning disorder. The patterns of $E_{x x}(x, y)$ are broad, while $E_{y y}(x, y)$ are modulated finer in enlarged imaging (b) and (c). The standard deviations of $E_{x x}(x, y)$ and $E_{y y}(x, y)$ given in the upper left corners of the maps are within a range $\leq 0.15 \%$, which is regarded as the extent of disturbance in strain measurements by the scanning disorder. 


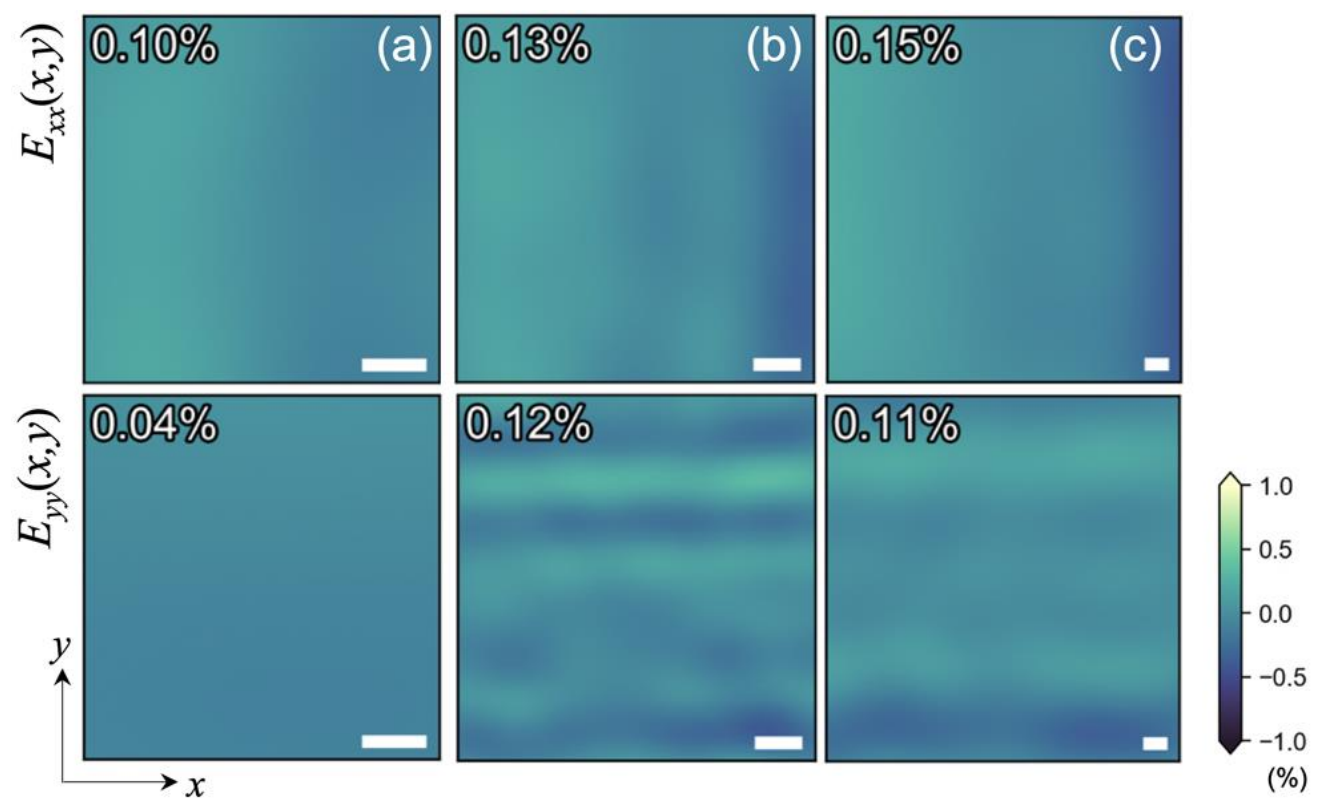

Figure S5. Maps of $E_{x x}(x, y)$ and $E_{y y}(x, y)$ or imaging magnifications and sizes of $\times 15 \mathrm{M}$ and $(512)^{2}$ pixels $(\mathrm{a}), \times 12 \mathrm{M}$ and $(1024)^{2}$ pixels $(\mathrm{b})$, and $\times 6 \mathrm{M}$ and $(2048)^{2}$ pixels $(\mathrm{c})$. The standard deviations are given at the upper left corner of each map. White scale bars at the lower right corners are $2 \mathrm{~nm}$.

\section{Details of molecular dynamics (MD) simulations}

Structural relaxations in MD simulations were conducted using the software LAMMPS ${ }^{8}$ with an embedded atom method (EAM) potential proposed by Pun et. al. ${ }^{9}$ for an Au crystal. Various structural properties have been reasonably reproduced as shown in Table S1, where the lattice constant $a_{0}$, elastic stiffness constants $C_{11}, C_{12}$, and $C_{44}$, elastic anisotropy ${ }^{10} A=$ $2 C_{44} /\left(C_{11}-C_{12}\right)$ and surface energies $\gamma_{111}, \gamma_{100}$, and $\gamma_{110}$ for $\{111\},\{100\}$, and $\{110\}$ are compared with the results of the density functional theory (DFT) ${ }^{11,12}$ and the experimental ${ }^{13,14}$ data. 
Table S1. Properties of crystalline gold derived by the EAM potential, DFT calculations, and experiments compared in terms of the lattice constant, elastic parameters, and surface energies

\begin{tabular}{ccccccccc}
\hline & $\begin{array}{c}\text { Lattice } \\
\text { constant }\end{array}$ & \multicolumn{2}{c}{ Bulk constants $(\mathrm{GPa})$} & \multicolumn{2}{c}{$\begin{array}{c}\text { Elastic } \\
\text { Anisotropy }\end{array}$} & \multicolumn{3}{c}{ Surface energy $\left(\mathrm{J} / \mathrm{m}^{2}\right)$} \\
& $a_{0}(\mathrm{pm})$ & $C_{11}$ & $C_{12}$ & $C_{44}$ & $A$ & $\gamma_{111}$ & $\gamma_{100}$ & $\gamma_{110}$ \\
\hline EAM $^{\mathrm{a}}$ & 407.8 & 205.4 & 167.8 & 48.8 & 2.56 & 1.29 & 1.33 & 1.42 \\
DFT & $407^{\mathrm{b}}$ & $202.1^{\mathrm{b}}$ & $174.2^{\mathrm{b}}$ & $37.9^{\mathrm{b}}$ & 2.72 & $1.28^{\mathrm{c}}$ & $1.63^{\mathrm{c}}$ & $1.70^{\mathrm{c}}$ \\
Experimental & $407.8^{\mathrm{d}}$ & $191^{\mathrm{d}}$ & $162^{\mathrm{d}}$ & $42.2^{\mathrm{d}}$ & 2.91 & $1.50^{\mathrm{e}}$ & & \\
\hline
\end{tabular}

${ }^{\mathrm{a}} \operatorname{Ref} 9,{ }^{\mathrm{b}} \operatorname{Ref} 11,{ }^{\mathrm{c}} \operatorname{Ref} 12,{ }^{\mathrm{d}} \operatorname{Ref} 13,{ }^{\mathrm{e}} \operatorname{Ref} 14$

The atomic models of nanoparticles are shown in Figure S6. As briefly introduced in the main text, MDrod models resembled in shape and size of the nanoparticles observed in the experiments. MDrod models consist of a cylinder capped with hemispherical cap ends. The cylindrical body is partially cut by $\{100\}$ and $\{110\}$ facets with considering stable facet condition of $\mathrm{Au}$. In contrast, MDsph models were in the form of spheroids with the corresponding sizes. The nanosphere NP1.0 is slightly faceted to $\{110\}$ and $\{111\}$ from a sphere to resemble the experimental observation.

The models were at first relaxed by minimizing the total energy at $0 \mathrm{~K}$. Random velocities were then given to atoms in the models corresponding to $600 \mathrm{~K}$. When the atoms lost kinetic energies moving to their new average positions, the systems were eventually equilibrated to 300 $\mathrm{K}$. The above relaxation procedure was carried out in the microcanonical (NVE) ensemble using a timestep of $1 \mathrm{fs}$. After the systems reached thermal equilibrium at $300 \mathrm{~K}$, the simulations were further run for another $600 \mathrm{ps}$ with a timestep of $1 \mathrm{fs}$ in the canonical (NVT) ensemble. A NoseHoover thermostat with a relaxation constant of $10 \mathrm{ps}$ was used to control the temperature. The atom positions were determined by averaged over the last 200 ps to exclude any possible memories on initial conditions. 

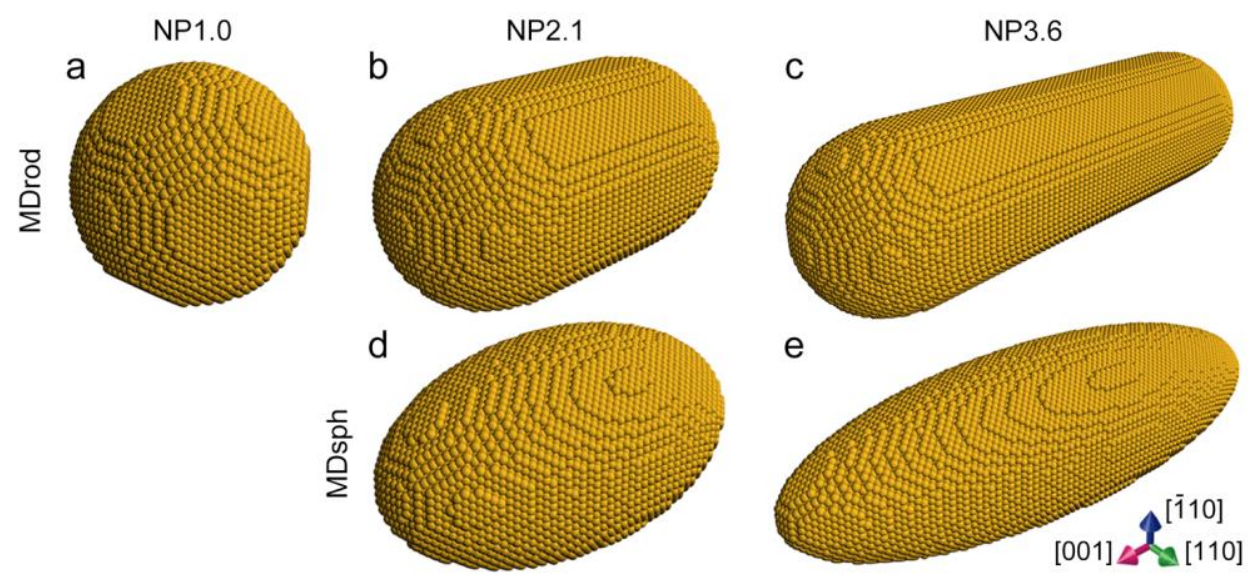

Figure S6. Atomic models relaxed in MD simulations. A sphere with the $\{111\}$ facets (a), rods consist of cylindrical bodies and hemispherical caps with the aspect ratios of 2.1 (b) and 3.6 (c), and spheroids with the aspect ratios of 2.1 (d) and 3.6 (e). The models are called as MDrod (a-c) and MDsph (d,e). The crystalographic orientations are indicated by arrows.

\section{References}

(1) Sato, Y.; Miyauchi, R.; Aoki, M.; Fujinaka, S.; Teranishi, R.; Kaneko, K. Large Electric-FieldInduced Strain Close to the Surface in Barium Titanate Studied by Atomic-Scale in Situ Electron Microscopy. Phys. status solidi-Rapid Res. Lett. 2020, 14 (1), 1900488. https://doi.org/10.1002/pssr.201900488.

(2) Fujinaka, S.; Sato, Y.; Teranishi, R.; Kaneko, K. Understanding of Scanning-System Distortions of Atomic-Scale Scanning Transmission Electron Microscopy Images for Accurate Lattice Parameter Measurements. J. Mater. Sci. 2020, 55 (19), 8123-8133. https://doi.org/10.1007/s10853-020-04602-w.

(3) Schmidbauer, M.; Kwasniewski, A.; Schwarzkopf, J. High-Precision Absolute Lattice Parameter Determination of $\mathrm{SrTiO}_{3}, \mathrm{DyScO}_{3}$ and $\mathrm{NdGaO}_{3}$ Single Crystals. Acta Crystallogr. Sect. B Struct. Sci. 2012, 68 (1), 8-14. https://doi.org/10.1107/S0108768111046738.

(4) Rasmussen, C. E.; Williams, C. K. I. Gaussian Processes for Machine Learning; The MIT Press: Cambridge, 2006.

(5) Bishop, C. M. Pattern Recognition and Machine Learning; Springer-Verlag: New York, 2006.

(6) McHutchon, A. Differentiating Gaussian Processes http://mlg.eng.cam.ac.uk/mchutchon/DifferentiatingGPs.pdf (accessed Jun 9, 2019). 
(7) Hines, T. T.; Hetland, E. A. Revealing Transient Strain in Geodetic Data with Gaussian Process Regression. Geophys. J. Int. 2017, 212 (3), 2116-2130. https://doi.org/10.1093/gji/ggx525.

(8) Plimpton, S. Fast Parallel Algorithms for Short-Range Molecular Dynamics. J. Comput. Phys. 1995, 117 (1), 1-19. https://doi.org/10.1006/jcph.1995.1039.

(9) Pun, G. P. P. 2017--Purja-Pun-G-P--Au https://www.ctcms.nist.gov/potentials/system/Au/ (accessed Aug 14, 2018).

(10) Chung, D. H.; Buessem, W. R. The Elastic Anisotropy of Crystals. J. Appl. Phys. 1967, 38 (5), 2010-2012. https://doi.org/10.1063/1.1709819.

(11) Wang, H.; Li, M. Ab Initio Calculations of Second-, Third-, and Fourth-Order Elastic Constants for Single Crystals. Phys. Rev. B - Condens. Matter Mater. Phys. 2009, 79 (22), 1-10. https://doi.org/10.1103/PhysRevB.79.224102.

(12) Vitos, L.; Ruban, A. V; Skriver, H. L.; Kolla, J. The Surface Energy of Metals. 1998, 411, 186202.

(13) Springer Handbook of Condensed Matter and Materials Data; Martienssen, W., Warlimont, H., Eds.; Springer Berlin Heidelberg: Berlin, Heidelberg, 2005; Vol. 66. https://doi.org/10.1007/3540-30437-1.

(14) Tyson, W. R.; Miller, W. A. Surface Free Energies of Solid Metals: Estimation from Liquid Surface Tension Measurements. Surf. Sci. 1977, 62 (1), 267-276. https://doi.org/10.1016/00396028(77)90442-3. 